\title{
Preliminary Comminications
}

\section{Simultaneous Umbilical Arteriovenous Exchange Transfusion}

\section{Brit. med. F., 1966, 2, 743-745}

The role of neonatal exchange transfusion in the prevention of kernicterus remains of paramount importance despite prenatal transfusions and premature delivery, and according to Mollison (1961) it carries a mortality of $1-5 \%$. In this paper we present a continuous-drip technique of exchange transfusion which we hope will carry a lesser mortality, and which has several advantages over the conventional intermittent-injection-andwithdrawal technique. An umbilical artery has been catheterized at varying times up to four days after birth, and exchange transfusion has been performed by spontaneous exsanguination from the artery and a gravity drip replacement via the umbilical vein.

\section{TEChNIQUe AND EQUIPMENT}

An exchange-transfusion kit containing the following is kept ready. (1) Two Plastipak 10-ml. syringes (Becton, Dickinson \& Co. Ltd.). (2) One 4.5 F.G. and one 9 F.G. soft polyvinyl Riplex umbilical catheter (W. Warne \& Co. Ltd.). (3) Two three-way " K.75" plastic stopcocks (Pharmaseal Laboratories Ltd.). (4) A metal base plate (Fig. 1). This is a modification

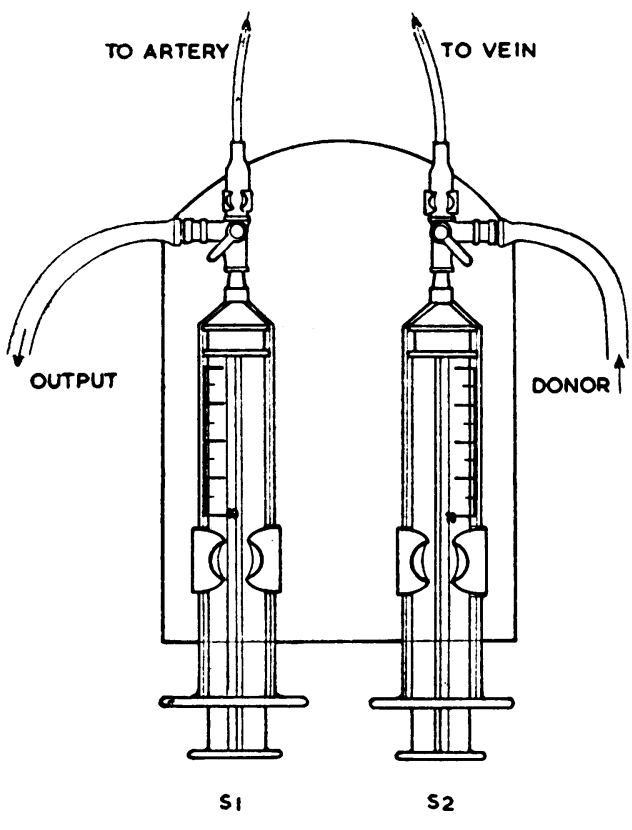

F1G. 1.-Stainless-steel plate with clips to fix syringes, stopcocks, and catheters.

of the original plate described by Simmons and Ata (1966). (5) A disposable infant's giving-set with a 30-ml. graduated chamber (Capon, Heaton \& Co. Ltd.). (6) An outlet cylinder graduated to $250 \mathrm{ml}$. (Fig. 2). The other equipment used consists of the usual surgical instruments, sterile sheets, etc.

The infant is placed in an incubator, preferably on a mobile tray which can be brought out. The bag or bottle of blood is hang on the stand by the side of the incubator and the outlet cylinder fixed on the bottom of the same stand. The catheters, syringes, and plastic taps are fixed on the base plate as in Fig. 1. After cleaning the umbilicus and the periumbilical skin, the cord is cut fairly short to a 1-cm. stump. The two arteries and the vein are identified. Dried stumps may be moistened by warm saline, which also helps to open the arterial lumen. A 4.5 F.G. catheter filled with saline is gently introduced into the arterial lumen. As shown in Fig. 3, the umbilical arteries have a tortuous course in the cord, most of which we cut off, and then run a gently curved, intra-abdominal course in which no

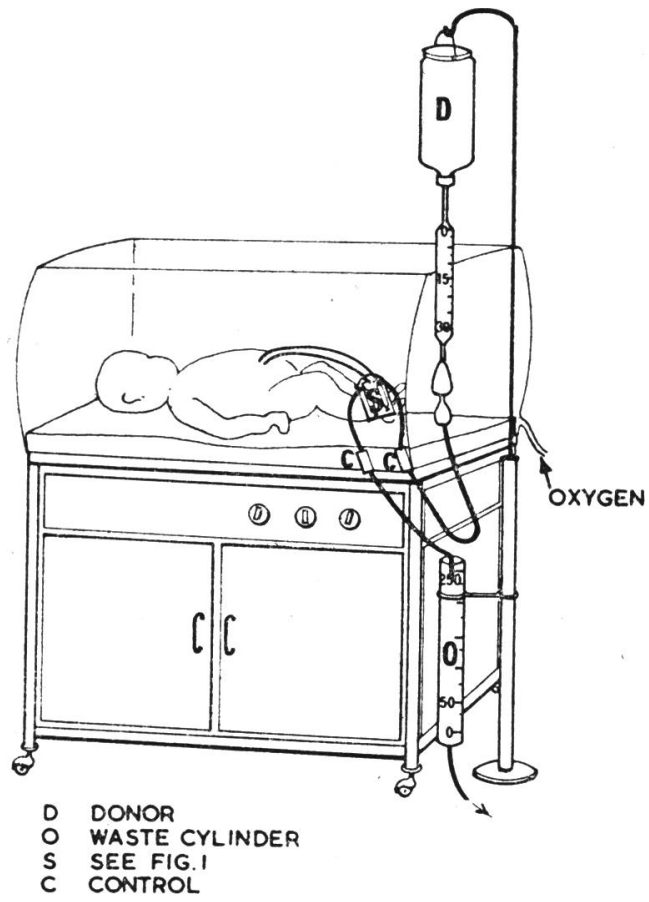

Fig. 2.-A diagrammatic representation of exchange transfusion in progress.

difficulty is encountered. Once in the arterial lumen the catheter is manœuvred downwards to pass for a distance of 6 in. $(15 \mathrm{~cm}$.), so that its tip lies in the abdominal aorta. When connected the arterial pulsation can be seen at the saline-blood junction in the catheter. Arterial catheterization has been found easier on the second or third day than immediately after birth because soft Wharton's jelly gives poor support to the artery in a fresh cord.

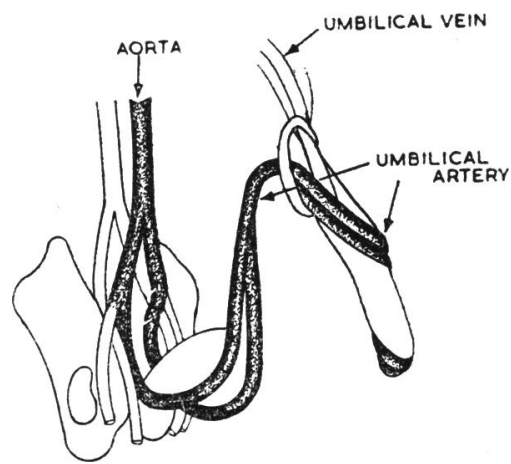

Fig. 3.-The umbilical vessels. 
The umbilical vein is catheterized with a 9 F.G. catheter. The infant is slid back into the incubator, and the donor blood (D) and outlet cylinder (O) are connected to venous and arterial catheters respectively (Fig. 2). The outlet tubing is flushed with heparin saline to prevent the blood from clotting in it. A specimen is drawn from the artery with syringe $S 1$ for various investigations-namely, haemoglobin, bilirubin, Coombs test, $p \mathrm{H}$, etc. - or for injecting any necessary drugs. Syringe $\mathrm{S} 2$ is used to prevent any air bubbles entering into the vein with blood. The taps are connected and the rate of exsanguination and replacement regulated and matched by control clips (C). The balance of volumes exchanged is checked frequently. The outlet cylinder is a long, thin column, 24 by 1 in. ( 61 by 2.5 $\mathrm{cm}$.), so that it offers accurate graduations up to $250 \mathrm{ml}$., and, once full, it has to be emptied into another reservoir. One pint $(570 \mathrm{ml}$.) of blood can be exchanged in half an hour if necessary by using a 6 F.G. catheter for the artery, but we prefer a rate of $5 \mathrm{ml} . / \mathrm{min}$. or one and a half hours for 1 pint, in general, and an even slower rate for particularly sick infants. If indicated, a second pint is used, and is preferably collected in a heparinized bag (Fenwal plastic packs). At the end of the transfusion the catheters are removed, when the artery may spurt and manual pressure is needed for a couple of minutes; very rarely is a stitch required.

\section{Preliminary Results}

So far 55 exchange transfusions on 40 infants have been performed. Many of these infants were very badly affected ; indeed, eight had been given prenatal transfusions. There was no case of kernicterus, but three infants died: one of the two with hydrops foetalis born alive was lost; an infant of 29 weeks' maturity died; and in the third infant, delivered by caesarean section for ante-partum haemorrhage, respiratory distress syndrome was the main cause of death. Death occurred more than 10 hours after transfusion in all three cases, and appeared unrelated to it. In no case did the umbilical arteries show any evidence of trauma or subintimal haemorrhage.

\section{Discussion}

A two-way technique of exchange transfusion involving withdrawal of blood from the sagittal sinus and replacement through a cannulated vein was proposed by Wallerstein (1946). Wiener and Wexler (1946) and Wiener et al. (1954) used a saphenous vein and the contralateral radial artery. This process cannot be used easily for repeat transfusions, and also carries risks from prolonged immobilization of the infant and local vascular impairment. One of us (C. A. H.) had tried umbilicalartery catheterization unsuccessfully in 1948. In recent years the artery has been used for sampling and infusions (Saling, 1959 ; Grychtolik and Kolrep, 1961 ; Ainsworth et al., 1963; Cottom, 1963 ; Layrisse and Linares, 1964). Saling (1959) catheterized the two umbilical arteries immediately after birth of infants at risk, and performed exchange transfusions, if indicated, later on. Layrisse and Linares (1964) used an apparatus for simultaneous manual injection of blood through an umbilical artery and withdrawal from the umbilical vein. By the present technique we have been able to catheterize the umbilical artery at varying times up to four days after birth, and it appears to have the following advantages:

1. No exacting physical work is needed after setting up the transfusion, and the operator is not tempted to empty a syringe forcefully. Elimination of jerky injections is likely to prevent undue strain on the myocardium, which may have been the cause of otherwise unexplained deaths.

2. The exsanguination continues as a drip under arterial pressure and the replacement as a gravity drip. Both run continuously and smoothly, and the operator, thus freed, can regulate the rate, watch the volumes, watch the oscilloscope, or make clinical and pathological assessments, including Astrup estimation of arterial blood $p \mathrm{H}$.

3. The apparatus is disposable, and technical difficulties due to sticky syringes, air leaks, etc., are completely avoided.

4. Because the inflow and outflow of blood are simultaneous, the time taken for the exchange transfusion can be halved or allowed to proceed at a much slower rate as desired. We prefer the latter, hoping to produce a better bilirubin clearance from the blood and tissues.

5. In a conventional alternative exchange transfusion there is a dead space of $1-1.5 \mathrm{ml}$. in the catheter, taps, and the syringe. This is occupied by the donor blood at the end of an injection phase, and is thrown out in the waste with the next withdrawal phase. However, by the present technique all the donor blood is infused into the infant's circulation. We estimated the amount of foetal haemoglobin before and after the exchange transfusion in a few cases. The average exchange transfusion of $500 \mathrm{ml}$. reduces the foetal haemoglobin level from about $75 \%$ to $8 \%$.

In most of the repeat transfusions the same artery was easily catheterized, but in some the other one was used. Generally there was a distinct improvement in the clinical state of the infant, and electrocardiographic changes were not observed. In two cases, in which transfusion was started by the old technique, extrasystoles were frequent in the injection phase; after changeover to the new technique no further disturbance was noted.

Although the exsanguination usually continues as a smooth drip, it may occasionally slow or stop because of clotting in the outflow tubing; a gentle suction with the syringe or flushing the outflow tubing with heparin saline removes the obstruction. Grychtolik and Kolrep (1961) described a case of gluteal gangrene from thrombosis, but if the catheter used is of plastic tubing and lies well in the aorta there is no danger of this. The bleeding at the time of removal of the arterial catheter is no problem if the 4.5 F.G. size is used, but in three cases where a 6 F.G. size was used prolonged manual pressure was required to stop the bleeding. Although this larger catheter gives a more rapid exchange-namely, 1 pint $(570 \mathrm{ml}$.) in 30 minutes-we prefer the 4.5 size.

A rate of infusion of $5 \mathrm{ml} . / \mathrm{min}$. is only a fraction of an infant's cardiac output, which averages $500 \mathrm{ml}$. The donor's blood mixes well with that of the infant in its cardiopulmonary vascular bed, and in our opinion offers a better mixing than that in the lower limbs when the artery is used for infusion and vein for withdrawal (as by Layrisse and Linares, 1964). We consider that, although the two arteries could be used to bypass the heart completely, it seems an unnecessary feat to combat the arterial pressure by gravity for infusion and also endanger the blood-supply to the lower limbs by two catheters in a tiny aorta compared with one catheter. We use the umbilical vein for infusion, since any complications-for example, portal hypertension and cirrhosis (Tizard, 1962 ; Shaldon and Sherlock, 1962)-are extremely rare (Thompson and Sherlock, 1964).

\section{SUMMARY}

Fifty-five arteriovenous exchange transfusions have been carried out on 40 infants. Spontaneous exsanguination of the infant from a catheterized umbilical artery and gravity drip replacement through the umbilical vein offers a smooth continuous exchange. This eliminates the irregular inflow which is inevitable when blood is injected manually. The operator can regulate the rates of flow, watch the volumes exchanged and the oscilloscope, or make clinical or short biochemical assessments. The infants tolerated large volumes well, and the grunting and crying seen when intermittent-injection-andwithdrawal technique was used were no longer observed.

Our thanks are due to our resident pathologists, Dr. Bharti Joshi and Dr. Sheela Amin, for their unfailing cooperation; to Sister M. 
Barlee and her staff for their help; to our pharmacist, Miss $\mathbf{P}$. Reynolds, for the provision of plastic equipment; and to $\mathrm{Mr}$. A. Sellers for making special metal fittings.

M. ATA, M.B., M.R.C.P.ED., M.C.PATH.,

Senior Registrar, Group Laboratory, Lewisham Hospital. C. A. Holman, M.B., M.R.C.P., F.C.PATH.,

Group Pathologist, Group Laboratory, Lewisham Hospital.

REFERENCES Ainsworth, R. W., Gairdner, D., Rack, J. H., and Webb, M. (1963).
Lancet, 1, 445.
Cottom, D. (1963). Ibid., 1, 329.

Grychtolik, H., and Kolrep, W. (1961). Kinderärztl. Prax., 29, 11.

Layrisse, M., and Linares, J. (1964). Transfusion (Philad.), 4, 33.

Mollison, P. L., (1961). Blood Transfusion in Clinical Medicine, 3rd ed., p. 640.' Blackwell, Oxford.

Saling, E. (1959). Geburtsh. u. Frauenheilk., 19, 230.

Shaldon, S., and Sherlock, S. (1962). Lancet, 1, 63.

Simmons, N. A., and Ata, M. (1966). Ibid., 1, 766.

Thompson, E. N., and Sherlock, S. (1964). Quart. F. Med., 33, 465.

Tizard, J. P. M. (1962). Proc. roy. Soc. Med., 55, 772.

Wallerstein, H. (1946). Science, 103, 583.

Wiener, A. S., and Wexler, I. B. (1946). F. Lab. clin. Med., 31, 1016. and Brancato, G. J. (1954). F. Pediat., 45, 546.

\section{Medical Memoranda}

\section{Hypercalcaemia Associated with Adeno- carcinoma of Kidney without Demonstrable Bone Lesions}

Brit. med. F., 1966, 2, 745-746

Hypercalcaemia as a complication of malignant disease with bone involvement is well recognized (Gutman et al., 1936; Myers, 1960 ; Moses and Spencer, 1963 ; Spencer and Lewin, 1963 ; Watson, 1963). Hypercalcaemia due to malignant disease, but unexplained by bone involvement, was first noted by Albright and Reifenstein (1948). Their patient had a hypernephroma, with a large metastasis in the sacrum and ilium, associated with hypercalcaemia, hypophosphataemia, and a normal serum alkaline phosphatase. Irradiation of the metastasis caused a transient fall in the serum calcium. They believed that the bone involvement in their case was not sufficient to cause the hypercalcaemia and did not account for the hypophosphataemia. They postulated that the tumour was producing a parathormone-like substance. Plimpton and Gellhorn (1956) reviewed 10 cases of malignant disease with hypercalcaemia in the absence of bone involvement, including four cases of hypernephroma and one case each of carcinoma of ovary, carcinoma of uterus, anaplastic retroperitoneal carcinoma, Hodgkin's disease, reticulum-cell sarcoma, and carcinoma of bronchus. In one case, in which the biochemical abnormalities reverted to normal after extirpation of the tumour, recurrence of the tumour was accompanied by recurrence of hypercalcaemia.

Malignant disease presenting solely with symptoms referable to the associated hypercalcaemia may cause difficulty in the diagnosis of the underlying condition. In these cases a clinical state indistinguishable from primary hyperparathyroidism may present, with hypercalcaemia, hypercalciuria, hypophosphataemia and occasionally a raised alkaline phosphatase value (Plimpton and Gellhorn, 1956 ; Alanis and Flanagan, 1959 ; Borm, 1961 ; Case Records, 1961 ; Goldberg et al., 1964 ; Svane, 1964 ; Snedecor and Baker, 1964 ; Tremblay and Ansell, 1964). Severe symptoms due to hypercalcaemia-thirst, polyuria, vomiting, lassitude, loss of weight, and mental disturbance-have led to a diagnosis of primary hyperparathyroidism and neck exploration in cases of otherwise occult carcinoma of oesophagus, bronchus, pancreas, endometrium, and ovary (Plimpton and Gellhorn, 1956 ; Goldberg et al., 1964 ; Snedecor and Baker, 1964).

Disease associated with calcium hyperabsorptive states, or bone-dissolution states, may present with the clinical picture of hypercalcaemia, and special investigations often fail to elucidate the nature of the problem. A review of the use of (1) measurement of tubular reabsorption of phosphorus, (2) response to intravenous calcium, and (3) response of serum calcium level to high doses of steroids, revealed their limitations in the differential diagnosis of hypercalcaemia (Thomas et al., 1958).

In the following case of carcinoma of the renal parenchyma the picture was confused by the symptoms of the associated hypercalcaemia.

\section{CASE REPORT}

A 55-year-old wood-machinist was first seen in another hospital in April 1963, complaining of weakness and vomiting of two months' duration. Investigations revealed a raised erythrocyte sedimentation rate, hypercalcaemia, raised alkaline phosphatase values, and a slight increase in plasma cells in the bone-marrow aspirate. A diagnosis of multiple myelomatosis was made and he was treated with melphalan, but with no improvement. He developed polyuria and pain in the shoulders, and was referred to the Royal Marsden Hospital for radiotherapy to relieve the pain.

In November 1961 he had had an episode of painless haematuria ; cystoscopy and intravenous pyelography were then thought to show nothing abnormal, and the haematuria was attributed to benign prostatic hyperplasia. $\mathrm{He}$ had no further haematuria or other urinary symptoms.

On admission to the Royal Marsden Hospital he was depressed and slow in mental reaction and at times appeared not to be in touch with his surroundings. Clinical examination revealed nothing further of note.

Investigations.- $\mathrm{Hb} 67 \%$; E.S.R. $28 \mathrm{~mm} . / \mathrm{hr}$.; W.B.C. 10,700 normal differential. Mid-stream specimen of urine showed a trace of protein, occasional R.B.C.s, no Bence Jones protein. Papanicolaou's stain showed no malignant cells in three specimens of urine. Blood urea was $32 \mathrm{mg} . / 100 \mathrm{ml}$. Serum calcium was $12.5 \mathrm{mg} . / 100 \mathrm{ml}$. ; phosphorus $2.3 \mathrm{mg} . / 100 \mathrm{ml}$, and alkaline phosphatase $39 \mathrm{KA}$. units: the subsequent behaviour of these values in response to steroids and nephrectomy is shown in Fig. 1. On three occasions the 24-hour urinary excretion of calcium was 480,800 , and $300 \mathrm{mg}$., and of phosphorus 570,900 , and $650 \mathrm{mg}$. The phosphorus clearance was within normal limits when related to the creatinine clearance. Steroid suppression test: $40 \mathrm{mg}$. of prednisolone daily for one week resulted in a decrease in serum calcium but no change in the urinary calcium excretion. Bone-marrow aspirate showed an excess of plasma cells. Skeletal survey revealed degenerative changes in the spine but no other abnormalities; the lamina dura were intact. I.V.P. showed a deformity in the upper pole of the left kidney which looked like a tumour on arteriography.

On 28 February 1964 a left nephrectomy was performed. Histology revealed a moderately well differentiated adenocarcinoma of the kidney (Fig. 2). Three days post-operatively the serum calcium was normal and the alkaline phosphatase gradually returned to a normal value. Seventeen months post-operatively the patient was well, with normal serum calcium values; and a bone-marrow aspirate performed two months post-operatively was considered to be normal. His hypercalciuria persisted for some time, and two months post-operatively values of 600 and $1,000 \mathrm{mg}$. in 24 hours were obtained. Seventeen months post-operatively the 24-hour 